\title{
THE INFLUENCE OF SLOW RECOVERY INSOLE ON PLANTAR PRESSURE AND CONTACT AREA DURING WALKING
}

\author{
ROOZBEH NAEMI*, KIMBERLEY LINYARD-TOUGH, \\ AOIFE HEALY and NACHIAPPAN CHOCKALINGAM \\ Center for Sport, Health and Exercise Research \\ Faculty of Health Science, Staffordshire University \\ Science Center, Leek Road, Stoke-on-Trent ST4 2DE, UK \\ *r.naemi@staffs.ac.uk
}

Received 28 January 2015

Accepted 3 February 2015

Published

\begin{abstract}
Plantar pressure assessment is commonly used as a tool to assess the efficacy of insoles in reducing the risk of mechanical trauma to the plantar soft tissue during walking gait. The slow rebound (SR) Poron insole is intended to provide a custom fit to the foot and is believed to be superior in increasing the contact area and consequently reducing the contact pressure compared to a normal Poron (NP) insole. The aim of this study was to compare the effectiveness of $\mathrm{SR}$ or NP versus an ethylene vinyl acetate (EV) insole in increasing the contact area $(\mathrm{CA})$, and in reducing the contact pressure $(\mathrm{CP})$ at different regions of the foot during walking. Plantar pressure data was collected from nine healthy individuals during walking using commercially available in-shoe plantar pressure sensors. Although, the NP insole significantly increased the $\mathrm{CA}$ and decreased the $\mathrm{CP}$ on the entire foot compared to the $\mathrm{EV}$, there was no significant change in $\mathrm{CP}$ or $\mathrm{CA}$ at any region of the foot in any of the tested insoles. CP showed a positive significant correlation with CA at heel, hallux and heel center in all three insoles. The expected significant negative correlation between regional CA and CP was not observed.
\end{abstract}

Keywords: Plantar pressure; insole material; slow rebound; foot regions; Poron®.

\section{Introduction}

Plantar pressure assessment has been frequently used to assess the insole's effectiveness in reducing the risk factors associated with soft tissue mechanical trauma during weight bearing activities of daily living. Conformity results in an increase in the contact area between the insole and the foot. Therefore, this is believed to result in a reduction of plantar pressure by distributing the force over a larger area. While high plantar pressure can be relieved by customized insoles that closely fit to the shape of the foot, ${ }^{1}$ it has also been shown that the plantar pressure parameters can

*Corresponding author. 


\section{R. Naemi et al.}

also be altered using a flat insole or a footbed that can conform to match the shape or the profile of the plantar surface of the foot. ${ }^{2,3}$

A material known as Poron® (Rogers Corporation) is recognized as being effective in reducing plantar pressure in diabetic neuropathic patients. ${ }^{4,5} \mathrm{~A}$ variety of Poron materials including a normal (Poron 4708), and a slow rebound variation (Poron 96) are commonly used in clinical practice. Compared to a normal Poron (NP), the slow rebound (SR) Poron is claimed to result in a custom fit during usage and is considered to be more effective in increasing the contact area and in reducing the interface pressure during usage.

In a previous study ${ }^{6}$ a significant difference was reported when the forefoot peak plantar pressure was compared for a SR Poron against a NP insole during walking. However, there is a paucity of studies which consider the effect of the two variations of Poron insole on contact area (CA) and contact pressure (CP) across different regions of the foot.

Furthermore, despite the fact that an increase in CA is believed to reduce the $\mathrm{CP}$, such a relationship has not been previously investigated across different regions of the foot. Such investigation could shed more light on the efficacy of SR Poron compared to a NP and in understanding the mechanism by which the plantar pressure parameters are affected during walking.

\section{Aim}

This study is aimed at investigating the effectiveness of NP and SR Poron insoles on the $\mathrm{CA}$, and $\mathrm{CP}$ at different regions of the foot during walking, and to assess whether the increase in the $\mathrm{CA}$ results in a decrease in $\mathrm{CP}$ in any of the tested insoles.

\section{Method}

Nine healthy individuals (five females and four males) with body mass $(76.8 \pm 24.4 \mathrm{~kg})$ and shoe UK size $(8 \pm 1)$ participated in this study. Appropriate ethical approval was granted from the Faculty's Ethics Committee.

The SR and NP flat insoles had $6.35 \mathrm{~mm}$ thickness with a Shore O hardness of 18 (Fig. 1). The $6 \mathrm{~mm}$ low density ethylene vinyl acetate (EVA) flat insole had Shore A hardness of $25 /$ shore $\mathrm{O}$ hardness $=35$ and was used as the control (Fig. 1). All insoles were tested in a Plimsoll (Fig. 1).

A commercially available in-shoe pressure measurement system (F-scan, Tekscan Inc., USA) was used to measure the interface pressure between the insole and the plantar surface of the foot, while the participant walked across the lab over a $10 \mathrm{~m}$ walkway at their preferred speed $(1.3 \pm 0.2 \mathrm{~m} / \mathrm{s})$. Plantar pressure data was recorded from each foot over five consecutive stance phases during each trial. The data was collected over five trials in each insole condition: The EV (control), SR Poron and the NP. A template was designed to divide the foot into 10 different 


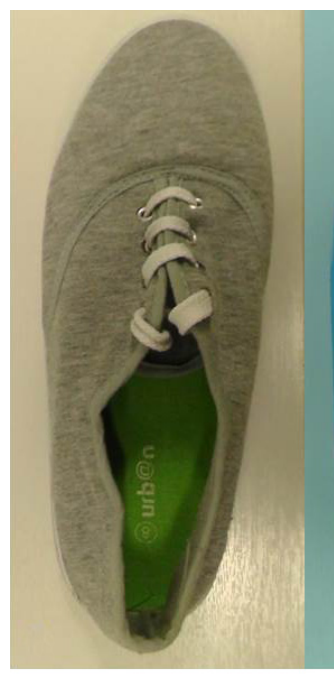

(a)

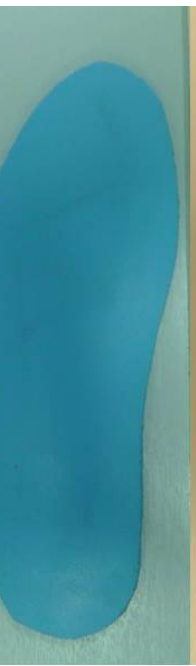

(b)

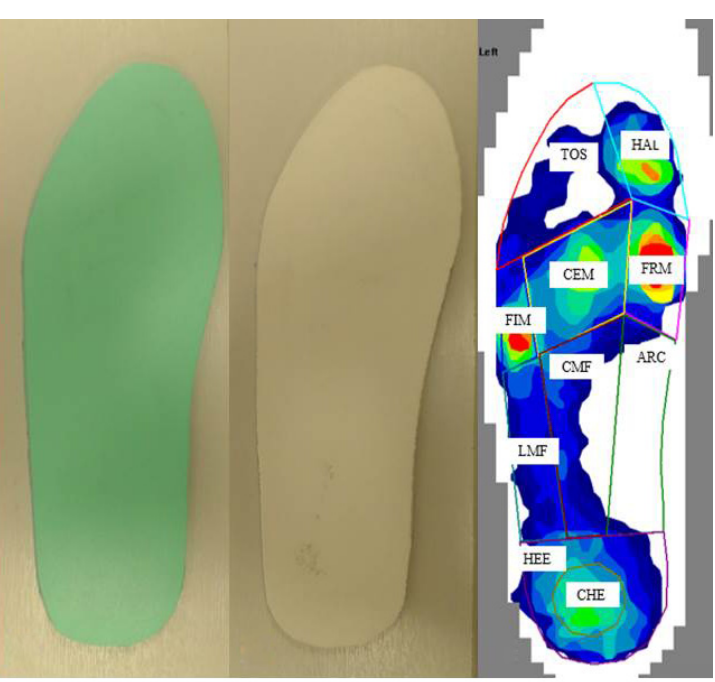

(c) (d) (e)

Fig. 1. (Color online) From left to right: (a) Plimsoll, (b) Normal Poron (NP) insole, (c) Slow Rebound (SR) Poron insole, (d) EVA (EV) insole and (e) the plantar pressure profile together with the template created to assess the plantar pressure parameters at 10 different regions underneath the foot.

regions, including underneath the arch (ARC), center of the midfoot (CMF), center of heel (CHE), Hallux (HAL), heel (HEE), first Metatarsophalangeal joint (FRM), central Metatarsophalangeal joints (CEM), fifth Metatarsophalangeal joint (FIM), and lateral midfoot (LMF) and toes (TOS) (Fig. 1). For each participant the CA and $\mathrm{CP}$ were averaged across 25 stance phases for the left and right foot separately. To identify if a significant difference in CA and CP between the three different insole conditions (SR), (NP) and (EV) across the entire foot or the 10 foot regions existed, a repeated measures ANOVA was performed. Furthermore, Pearson product-moment correlation was conducted to determine the relationship between the CA and $\mathrm{CP}$ at different regions of the foot when different types of insoles were used. All statistical tests were performed using SPSS (IBM Corp. Released 2013. IBM SPSS Statistics for Windows, Version 22.0. Armonk, NY: IBM Corp) software.

\section{Results}

\subsection{Significant differences $(\boldsymbol{p}<0.05)$}

$C A(S R$ versus $N P)$ : The $\mathrm{CA}$ at the $\mathrm{ARC}\left(\eta^{2}=0.409, p=0.020\right)$ and $\mathrm{CMF}$ $\left(\eta^{2}=0.719, p=0.021\right)$ in SR were significantly higher compared to NP condition (Fig. 2).

$C A$ (SR versus $E V)$ : The $\mathrm{CA}$ at the $\mathrm{ARC}\left(\eta^{2}=0.409, p=0.010\right), \mathrm{CMF}$ $\left(\eta^{2}=0.719, p=0.000\right)$ and LMF $\left(\eta^{2}=0.592, p=0.000\right)$ in SR were significantly higher compared to EV (Fig. 2). 


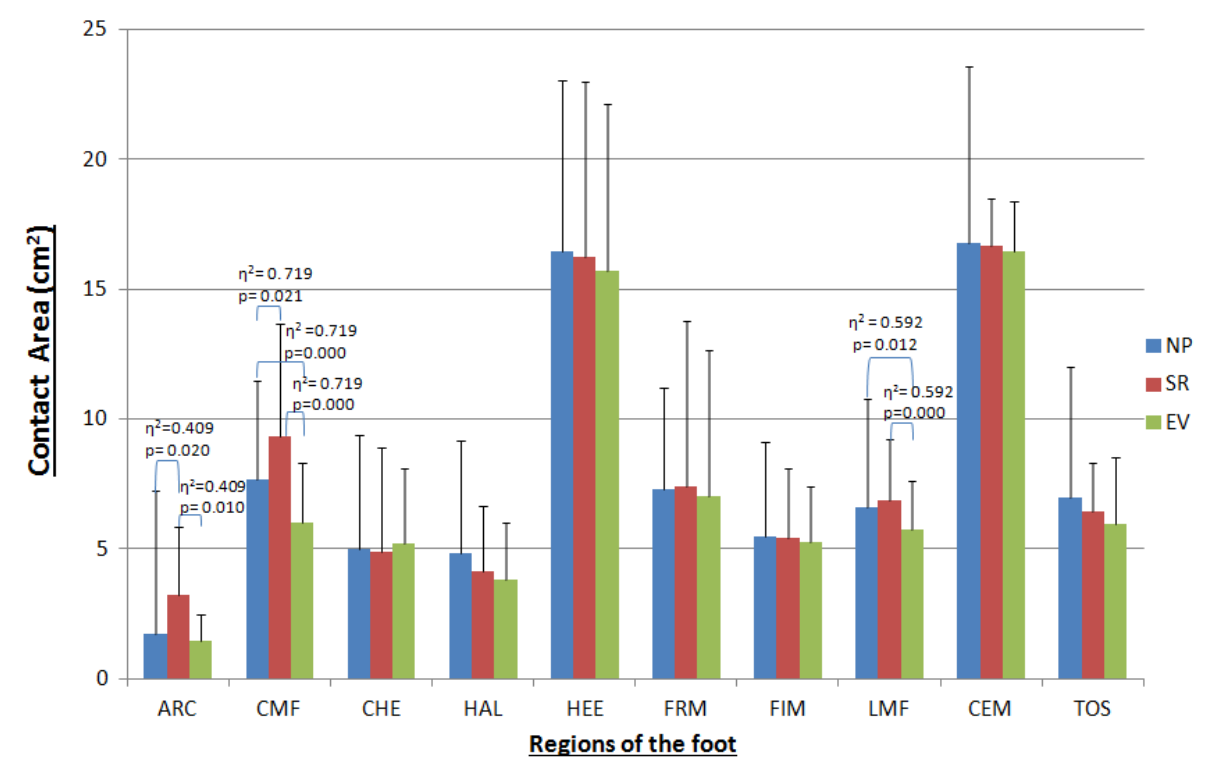

Fig. 2. (Color online) CA across different regions of the foot for SR, NP, and EV. Significant differences are shown with a bracket.

$C A$ (NP versus $E V)$ : There was a significant increase $\left(\eta^{2}=0.557, p=0.001\right)$ in the CA for the entire foot in NP $\left(77.10 \pm 12.98 \mathrm{~cm}^{2}\right)$ compared to EV $\left(70.19 \pm 11.97 \mathrm{~cm}^{2}\right)$, while $\operatorname{CMF}\left(\eta^{2}=0.719, p=0.000\right)$ and LMF $\left(\eta^{2}=0.592\right.$, $p=0.012$ ) had significantly higher CA in the NP compared to EV condition (Fig. 2).

$C P(S R$ versus $N P)$ : The $\mathrm{CP}$ at $\mathrm{ARC}$ region was significantly $\left(\eta^{2}=0.494\right.$, $p=0.010)$ higher in SR compared to the NP condition (Fig. 3).

$C P(S R$ versus $E V)$ : There was a significant increase in the $\mathrm{CP}$ in the $\mathrm{ARC}$ $\left(\eta^{2}=0.494, p=0.004\right), \operatorname{CMF}\left(\eta^{2}=0.318, p=0.042\right)$ and $\operatorname{LMF}\left(\eta^{2}=0.296\right.$, $p=0.049)$ regions in SR as compared to EV condition (Fig. 3).

$C P$ (NP versus $E V)$ : For the entire foot, there was a significant decrease $\left(\eta^{2}=0.386, \quad p=0.015\right)$ in $\mathrm{CP}$ in $\mathrm{NP}(65.01 \pm 27.16 \mathrm{kPa})$ compared to $\mathrm{EV}$ $(101.16 \pm 28.35 \mathrm{kPa})$ condition (Fig. 3).

\subsection{Significant relationships $(p<0.05)$}

$N P$ : There was a significant correlation between $\mathrm{CA}$ and $\mathrm{CP}$ in NP condition at CHE $(r=0.701, p=0.001)$, HAL $(r=0.597, p=0.009)$, HEE $(r=0.477$, $p=0.045)$, and FRM $(r=0.544, p=0.020)$ regions.

$S R$ : Significant correlations were found between CA and CP in SR condition at CHE $(r=0.734, p=0.001)$, HAL $(r=0.623, p=0.006)$, HEE $(r=0.500$, $p=0.035)$, and FIM $(r=0.652, p=0.003)$ regions.

$E V$ : It was found that $\mathrm{CA}$ and $\mathrm{CP}$ were significantly correlated in EV condition at $\mathrm{CHE}(r=0.734, p=0.001)$, HAL $(r=0.663, p=0.006)$, HEE $(r=0.592$, 
The Influence of Slow Recovery Insole on Plantar Pressure

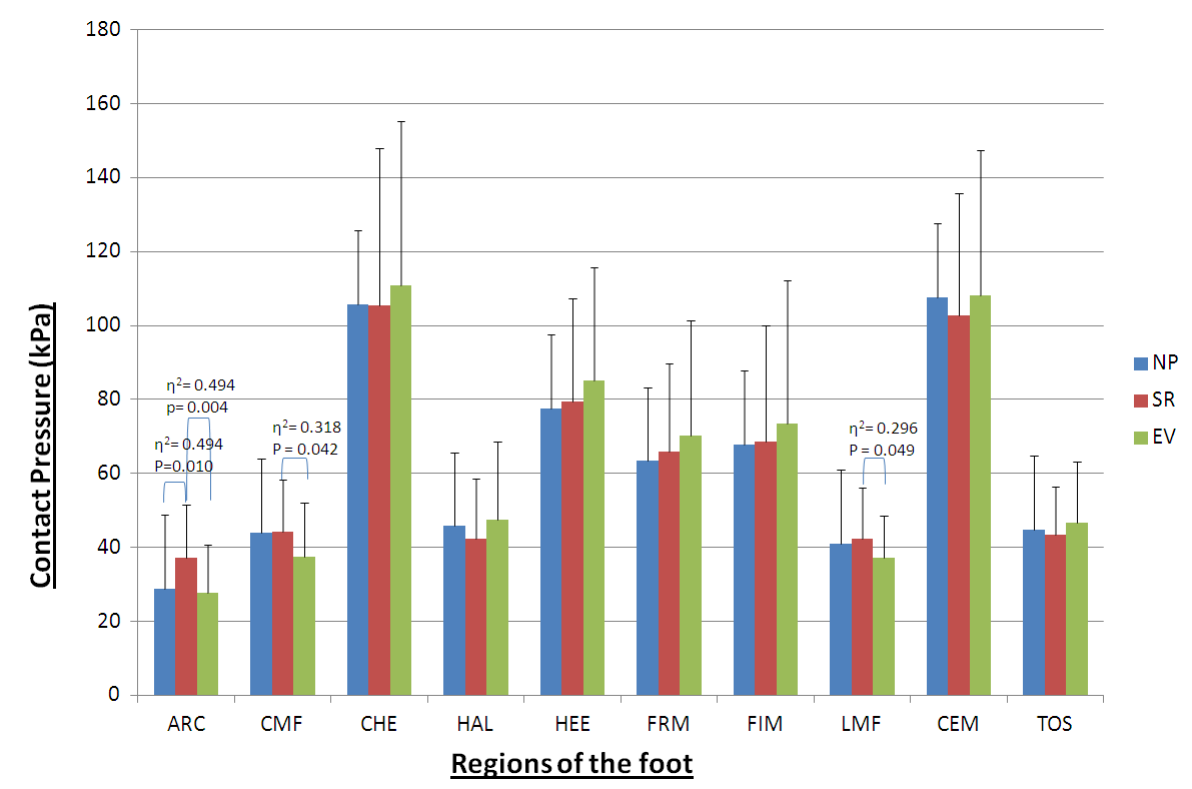

Fig. 3. (Color online) CP across different regions of the foot for SR, NP, and EV. Significant differences are shown with a bracket.

$p=0.010) \mathrm{FRM}(r=0.589, p=0.010)$, FIM $(r=0.507, p=0.032)$, and CEM $(r=0.469, p=0.50)$ regions.

\section{Discussion}

Both SR and NP, compared to EV, tend to significantly increase CA at CMF and LMF regions. While SR showed to have an increased CA at CMF compared to NP and significantly higher CA at ARC compared to both NP and EV, NP showed a significantly higher $\mathrm{CA}$ over the entire foot compared to the EV insole condition.

SR showed a significant increase in the $\mathrm{CP}$ at ARC area compared to both EV and NP conditions. Also the SR compared to EV condition seem to cause significantly higher pressure at CMF and LMF. This may have practical implications where increasing pressure at certain regions of the foot is not desirable.

When considering the entire foot, only NP insole significantly increased the CA and decreased the CP compared to EV insole. Tests of relationship showed significant and positive correlations between $\mathrm{CA}$ and $\mathrm{CP}$ in the HEE, HAL and CHE regions in all tested insoles. For other regions there was no consistent correlations between CA and CP that was valid across all three insole conditions. For example, in NP condition there was a significant positive correlation between $\mathrm{CP}$ and $\mathrm{CA}$ at FRM, and at FIM in SR condition. Also, positive significant correlations were observed at FRM, FIM, and CEM in EV condition. The fact that no negative correlation was observed between the $\mathrm{CA}$ and $\mathrm{CP}$ at any plantar region of the foot 


\section{R. Naemi et al.}

for any of the tested insoles, contradicted the expected decrease in $\mathrm{CP}$ as a result of the increase in CA. This can be attributed to the thickness of the insoles that were tested in this study and the possibility of these to have bottomed out during the stance phase of walking. This could have resulted in simultaneous increase in the pressure and contact area. More studies are needed in future to investigate the effect of insole thickness on the reported parameters in this study.

\section{Conclusion}

The results of this study indicated that only NP insole, when compared to the EV insole, increased the CA and decreased the CP on the entire plantar surface of the foot. SR showed to significantly increase $\mathrm{CA}$ at $\mathrm{ARC}$ and $\mathrm{CMF}$ regions compared to NP. The unexpected positive significant correlation between CA and CP were observed at the HEE, HAL and CHE regions for all tested insoles.

\section{Acknowledgment}

Funding from DiabSmart project is acknowledged. DiabSmart project was funded by the European Commission, Grant Agreement Number 285985, under Industry Academia Partnerships and Pathways (FP7-PEOPLE-2011-IAPP). This project has a focus on development of a new generation of Diabetic footwear using an integrated approach and Smart materials.

\section{References}

1. Bus S, Ulbrecht J, Cavanagh P, Pressure relief and load redistribution by custom-made insoles in diabetic patients with neuropathy and foot deformity, Clin Biomech 19:629638, 2004.

2. Tsung BY, Zhang M, Mak AF, Wong MW, Effectiveness of insoles on plantar pressure redistribution, J Rehabil Res De 41:767-768, 2004.

3. Healy A, Dunning D, Chocklingam N, Effects of insole material on lower limb kinematics and plantar pressure during treadmill walking, Prosthet Orthot Int 36:53-62, 2011.

4. Birke JA, Foto JG, Pfiefer LA, Effect of orthosis material hardness on walking pressure in high-risk diabetes patients, J Prosthet Orthot 11:43-46, 1999.

5. Burns J, Begg L, Vicaretti M, Comparison of orthotic materials on foot pain, comfort and plantar pressure in the neuroischemic diabetic foot: A case report, J Am Podiatr Med Assoc 98:143-148, 2008.

6. Tong JW, Ng EY, Preliminary investigation on the reduction of plantar loading pressure with different insole materials, The Foot 20:1-6, 2010. 